\title{
Velocity field of isolated turbulent puffs
}

\author{
E. Ghaem-Maghami ${ }^{1}$ and $\mathrm{H}$. Johari ${ }^{2, a)}$ \\ ${ }_{1}^{1}$ Machflow Energy, Inc., Worcester, Massachusetts 01610, USA \\ ${ }^{2}$ Department of Mechanical Engineering, California State University, Northridge, California 91330, USA
}

(Received 4 June 2010; accepted 23 September 2010; published online 4 November 2010)

\begin{abstract}
The velocity field of isolated turbulent puffs was measured using the particle image velocimetry technique and was compared with the steady jet flow field. Puffs were generated by injecting air through a $5 \mathrm{~mm}$ diameter nozzle into a flow chamber with a weak coflow. Isolated puffs with a Reynolds number of 5000 were examined in the range of 40-75 diameters downstream of the nozzle. The injection time was varied in order to assess the effects of injection volume and equivalent stroke ratio on the puff structure. The results from phase-locked measurements indicate that as the injection volume increased, puffs elongated in the axial direction and became similar to starting jets in the range considered. The largest scaled fluctuating velocities and turbulent shear stress within the puffs were twice the steady jet values. Inspection of the vorticity field revealed the presence of vorticity throughout the puff volume. Entrainment takes place on the portion of the puff closest to the nozzle and the entrainment rate is greater for the puffs with the smaller injection volume. This is consistent with the observations of rapid mixing and combustion of puffs in previous studies. (C) 2010 American Institute of Physics. [doi:10.1063/1.3504378]
\end{abstract}

\section{INTRODUCTION}

The entrainment and mixing associated with turbulent structures are not only of interest from a fundamental perspective but also have many practical applications. One of the most extensively investigated free shear flows in the past is the steady turbulent jet. On the other hand, much less is known about the mixing and structure of unsteady or pulsatile jet flows. Yet the various actuation mechanisms in modern flow control strategies create unsteady or intermittent jets. Moreover, pulsatile combustion has shown rapid fuel/air mixing, reduced exhaust emissions, and the potential for compact and economical combustors. ${ }^{1}$ Pulsatile nonreacting jets have also shown increased entrainment both in the near field and the far field. ${ }^{2-4}$ Fully modulated jets appear to have significantly increased entrainment even in the far field. ${ }^{3}$ Experiments on reacting, fully modulated pulsed jets have indicated significantly reduced flame lengths when injection times are short and there is little interaction among successive flame puffs. ${ }^{5,6}$ Under these conditions, the fuel jet consists of a sequence of distinct turbulent puffs. These puffs burn rapidly in comparison with a steady jet. It has been argued that the greater entrainment of isolated turbulent puffs, compared with a steady jet, is responsible for the observed short flame lengths. ${ }^{5,6}$ We are therefore interested in examining the velocity field and entrainment of isolated turbulent puffs in the far field. Previous works on turbulent puffs are briefly reviewed below.

A puff refers to a mass of turbulent fluid moving unsteadily through its surroundings with which it mixes readily. $^{7-9}$ The puff structure has been considered rather similar to a turbulent vortex ring with the exception that the

\footnotetext{
a) Author to whom correspondence should be addressed. Electronic mail: hjohari@csun.edu.
}

vorticity is spread throughout the puff volume. ${ }^{10}$ In fact, the scaling laws for the spreading and celerity of puffs and vortex rings are identical. ${ }^{7,8,10}$ In the far field, the puff width increases linearly with the distance traveled (from a virtual source) and the puff volume scales with the cube of puff width. The puff celerity scales with the inverse of the axial distance cubed. Sangras et al. ${ }^{11}$ measured the penetration and width of turbulent puffs in aqueous media by recording the visual outline of puffs at Reynolds numbers of $3-12 \times 10^{3}$, comparable to that in the present experiments. The penetration and width of puffs followed the self-similar scaling for distances greater than 20-30 source diameters. ${ }^{11}$ The mixing of isolated puffs has also been studied by the measurement of passive scalar concentration field within the puff. $^{12}$ Kovasznay et al. ${ }^{8}$ examined the flow field of a turbulent puff using the hot-wire anemometry technique. Their study focused on the puff celerity and the mean velocity in the near field (within the first ten diameters). The mean and fluctuating velocity of turbulent puffs have not been investigated in the far field in the past studies.

A turbulent puff, schematically shown in Fig. 1, may be generated by an impulsive injection of fluid through a nozzle. For stroke ratios (injected slug length $L$ over nozzle diameter $d$ ) of order one, a common vortex ring is generated. As the stroke ratio increases, the injected fluid eventually forms a puff in the far field. For very large stroke ratios, a starting jet is created. Volumetric arguments have been used to show that the flame length of an isolated burning puff scales with the cube root of the injected fuel volume. ${ }^{5}$ Following a similar reasoning, a dimensionless parameter $P$ based on the cube root of the puff volume $V_{o}$ has been developed to classify whether an impulsively initiated flow results in a puff or an intermittent jet (elongated structure) over a certain range in the far field. ${ }^{6}$ 


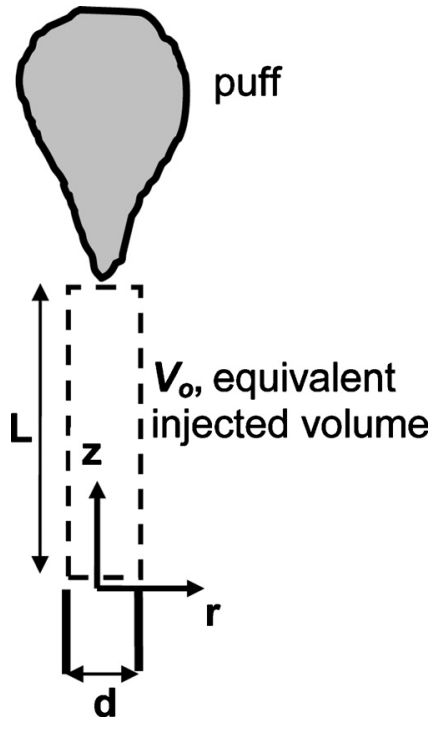

FIG. 1. Schematic of turbulent puff with the relevant injection parameters.

$$
P \equiv\left(4 V_{o} / \pi\right)^{1 / 3} / d=(L / d)^{1 / 3} .
$$

In effect, $P$ is the cube root of the injected fluid stroke ratio. Past experiments have shown that for $P \leq 8$, pufflike structures result in the range of axial distances up to $100 d^{6,11}$ For $P>11$, the flow is essentially a starting jet in the same range. Transitional, elongated flow structures are formed for $8<P<11$. Distinct vortex rings are found for stroke ratios of less than about 4 , corresponding to $P<1.6$. ${ }^{13}$

The primary objective of this study was to investigate the internal structure of isolated, constant density puffs and their entrainment characteristics. To accomplish these objectives, the velocity field associated with the far field of individual, nonreacting, turbulent puffs was measured using the planar particle image velocimetry (PIV) technique. The focus of the current work is on the gaseous puffs with $4 \leq P \leq 8$ at Reynolds number of 5000 and in the axial range of 40-75 nozzle diameters.

\section{EXPERIMENTAL TECHNIQUE}

The experimental apparatus consisted of a coflowing chamber with a square cross-section of $0.3 \times 0.3 \mathrm{~m}^{2}$. Three sides of the chamber were made from glass to allow optical access. A nozzle with an inner diameter $d=5 \mathrm{~mm}$ and placed in the center of the chamber delivered the puff flow. The coflow was uniform away from the intermediate vicinity of the nozzle and had a mean velocity corresponding to $0.5 \%$ of the nozzle velocity. The nozzle flow was controlled by a fast-response $(\sim 500 \mu \mathrm{s})$ solenoid valve actuated in an "onoff" (square wave) manner by an electronic control unit. The nozzle Reynolds number based on the average volume flow rate and diameter was set at 5000. The solenoid valve was pulsed at a sufficiently low frequency such that each puff cleared the test section completely before a subsequent puff was generated. The flow injection times ranged from $20 \mathrm{~ms}$ for the $P=4$ case to $161 \mathrm{~ms}$ for the $P=8$ case.

The instantaneous velocity field of each isolated puff was measured using a two-component PIV system. Laser
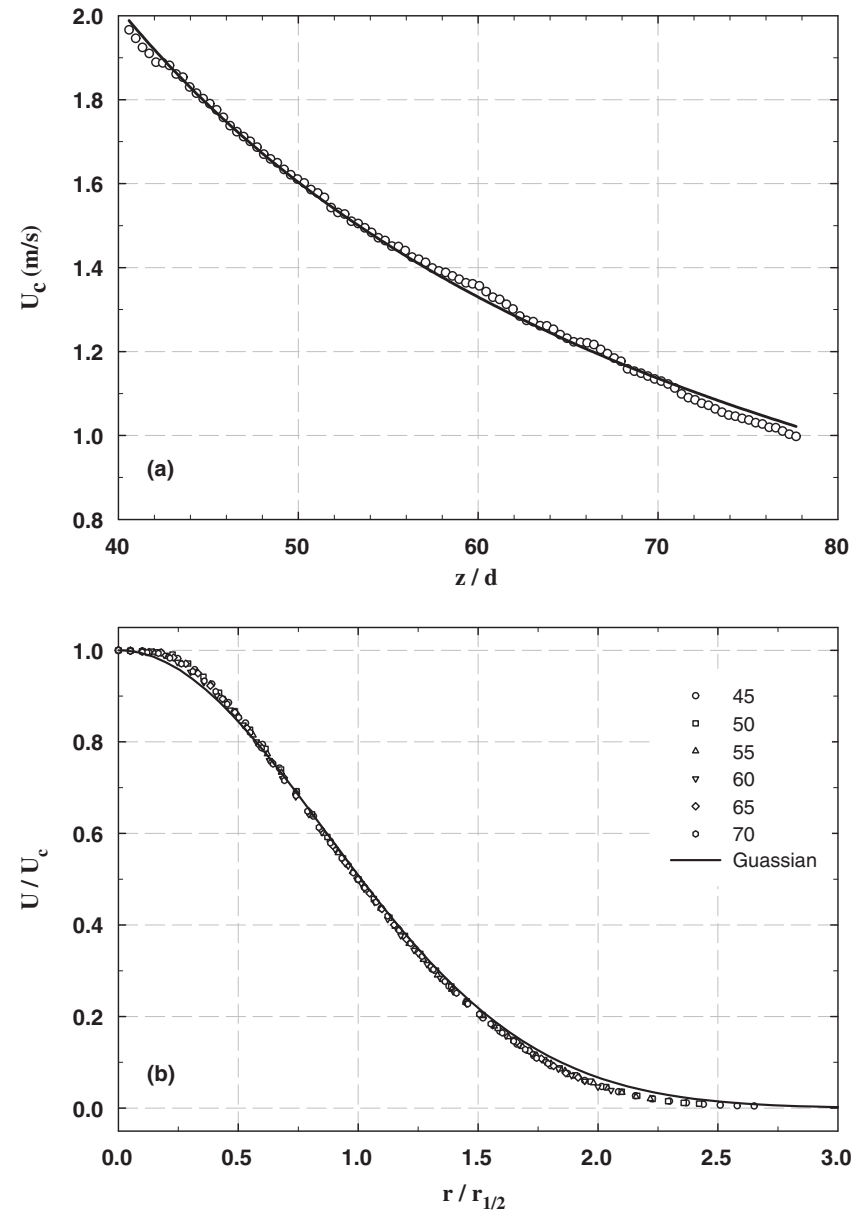

FIG. 2. Steady jet mean velocity. (a) Centerline velocity decay; the solid line is the best fit of Eq. (2). (b) Axial velocity profiles in similarity coordinates; the solid curve is the best-fit Gaussian curve.

pulses from a dual Nd:YAG laser system were directed through an optical setup with an adjustable focus and divergence to create a laser sheet for illumination purposes. The time separation between pulses was set depending on the puff velocity in the domain of interest. The flow field images were acquired with a CCD camera with a 1600 $\times 1200$ pixel resolution and a $50 \mathrm{~mm} f 1.8$ lens. For the present set of experiments, the coflow was seeded with aluminum oxide particles with a mean diameter of $9 \mu \mathrm{m}$. The entrainment of the coflow into the puff prior to the imaging region provided ample seeding for the PIV technique. The image acquisition was phase-locked to the valve pulsing signal with a time delay. The time delay was adjusted such that the puffs were positioned in the center of the image. The average uncertainty of the particle displacement and velocity measurements is estimated to be $\pm 1.3 \%$, based on the uncertainty of the correlation peak location and the average displacement. ${ }^{14} \mathrm{~A}$ set of runs were performed in which a steady jet was established in the test section and the jet data were used to verify the experimental technique. This data set is described in Sec. III.

For each case, 2000 instantaneous phase-locked velocity fields were ensemble averaged. In all cases, puffs were centered within the image in the range of $40 d-75 d$ in the axial direction. The velocity distribution within the averaged fields 

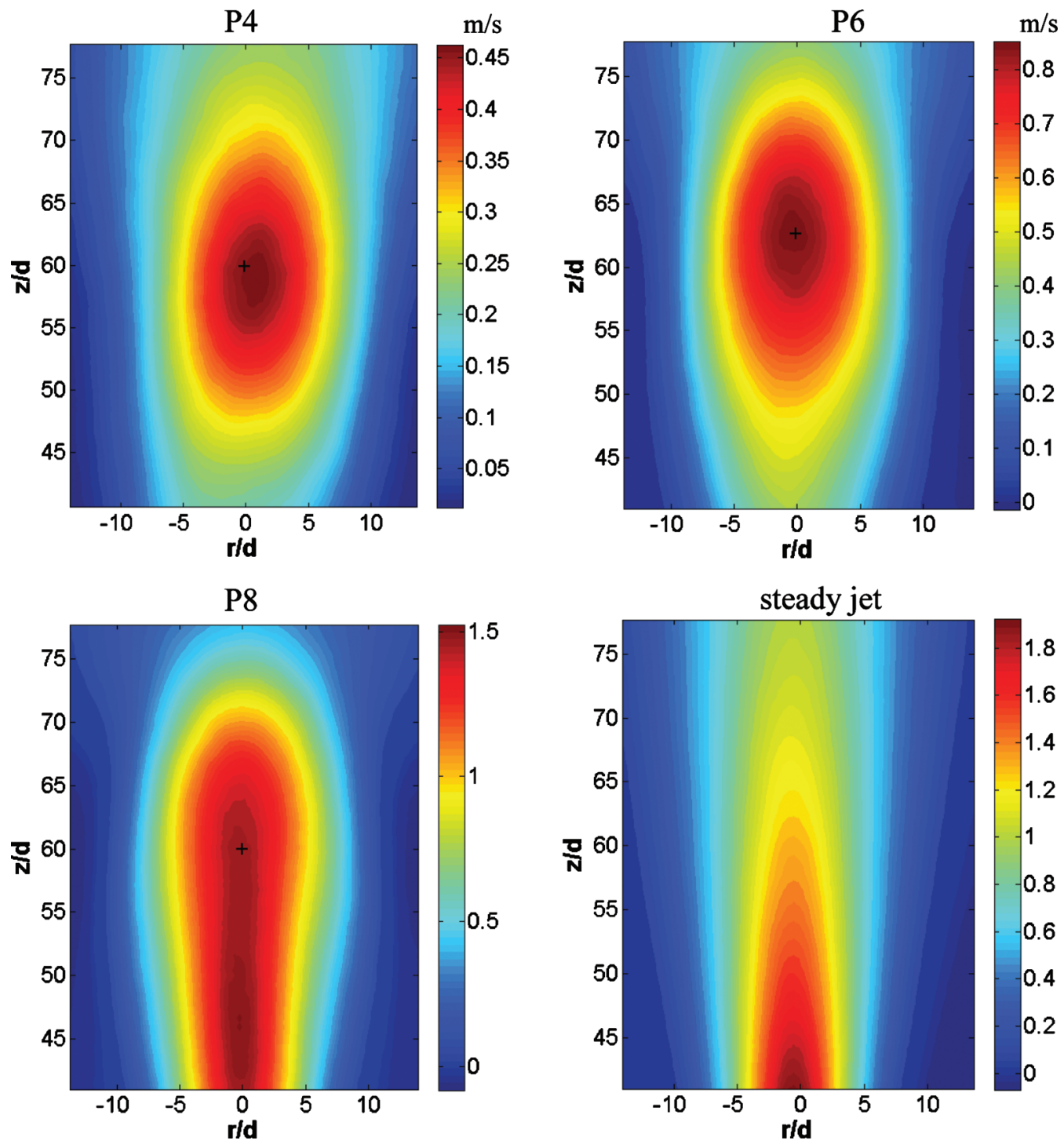

FIG. 3. (Color) Axial velocity contours for the puffs and the steady jet. The flow is from the bottom to the top. The plus sign denotes the location of puff center.

was examined as a function of the injection parameter $P$. The puff center $z_{c}$ was defined by the location of maximum velocity within the averaged field. Radial and axial velocity profiles as well as turbulent shear stress at the location of puff center were extracted and compared among the puffs and with the steady jet.

\section{RESULTS AND DISCUSSION}

\section{A. Steady jet}

To verify the experimental setup and the measurement technique, the mean velocity field of the steady jet resulting from continuous injection from the same setup was compared with the available data in the literature. The steady jet data were used also for comparison with the puff flow fields. The uncertainty of the axial velocity data presented here is $\pm 1.8 \%$. The decay of centerline velocity $U_{c}$ in the selfsimilar region of the jet can be described by

$$
\frac{U_{c}}{U_{o}}=B_{u} \frac{d}{z-z_{o}},
$$

where $U_{o}$ is the nozzle velocity and $z_{o}$ is the virtual origin. $B_{u}$ is the decay constant which, in general, has a value between 5 to 6 depending on the experimental setup and conditions. ${ }^{15-19}$ The fitting of the mean centerline velocity in Fig. 2(a) to Eq. (2) results in a decay constant of $B_{u}=5.2$ and a virtual origin of $z_{o}=1.4 d$. These values are consistent with the available data in the literature.

To determine whether the mean axial velocity $U$ has reached a self-similar state in the region investigated, $U / U_{c}$ is plotted against $r / r_{1 / 2}$ for six axial locations in Fig. 2(b). The half-radius $r_{1 / 2}$, the radial distance where the axial velocity is one half of the centerline value, was extracted from the mean velocity profile at each axial location. All the profiles within the experimental region collapse together, indi- 


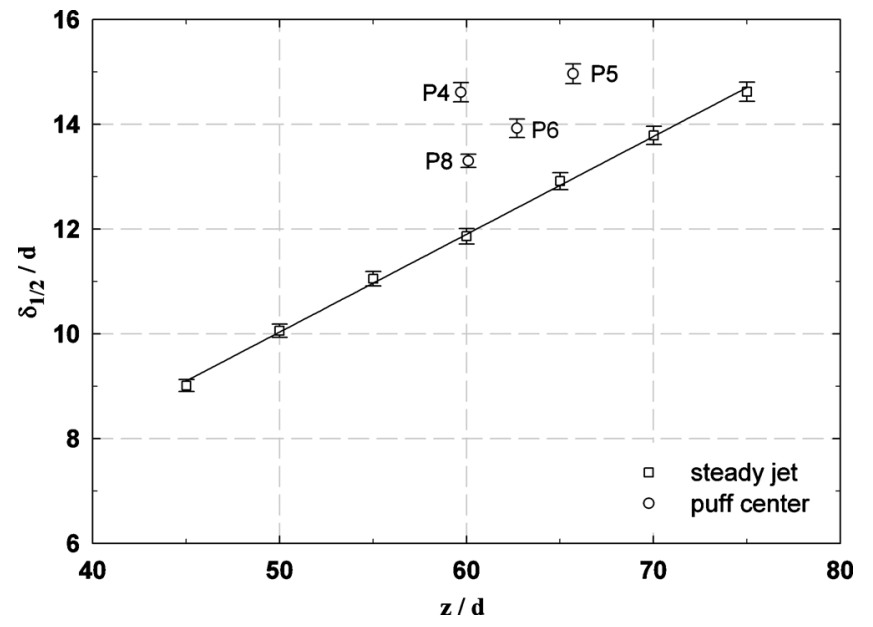

FIG. 4. Half-width of puffs and the steady jet.

cating that the mean jet velocity profiles are self-similar. These velocity profiles may be described by a Gaussian function as follows:

$$
U=U_{c} \exp \left[-k_{u}(r / z)^{2}\right] .
$$

A least-square fit to the data in Fig. 2(b) resulted in the fitting constant $k_{u}=76.9$. The experiments of Panchapakesan and Lumley $^{20}$ produced a value of $k_{u}=75.2$ at $\mathrm{Re}=1.1$ $\times 10^{4}$, whereas the direct numerical simulation study of Boersma et al. ${ }^{18}$ resulted in a value of $k_{u}=76.1$ at $\mathrm{Re}=2.4$ $\times 10^{3}$. Hence, the width of the mean velocity profile as indicated by $k_{u}$ in the present experiment is consistent with those in previous experimental and numerical studies. Aside from the mean velocity, the fluctuating velocity and turbulent shear stress profiles were also found to be comparable to the data in the literature. These profiles will be presented along with those from the isolated puffs in the subsequent sections. Thus, continuous injection in the present experimental setup created a self-similar steady jet with properties similar to the previous

studies.

\section{B. Puff mean velocity}

The phase-averaged axial velocity contours for $P=4,6$, and 8 puffs, and the steady jet are presented in Fig. 3. The puff center, denoted by the plus (+) symbol in the plots, was chosen as the location of maximum axial velocity. For the cases considered, the puff center was located at approximately $60 d$. The contour plots in Fig. 3 reveal that the puff geometry evolves from a prolate ellipsoid at $P=4$ to an elongated structure resembling a starting jet at $P=8$. The $P=5$ case was quite similar to the $P=4$ puff. The steady jet, in contrast, has an expanding conical geometry with the maximum velocity at the upstream end.

The puff half-radius $r_{1 / 2}$ was defined as the radial extent where the axial velocity decreases to one half of the maximum value at the puff center. To account for any asymmetry between the two sides of the data set, the puff half-width $\delta_{1 / 2}$ was extracted from the average axial velocity profiles. The puff half-radius $r_{1 / 2}$ was then chosen as one half of the half-

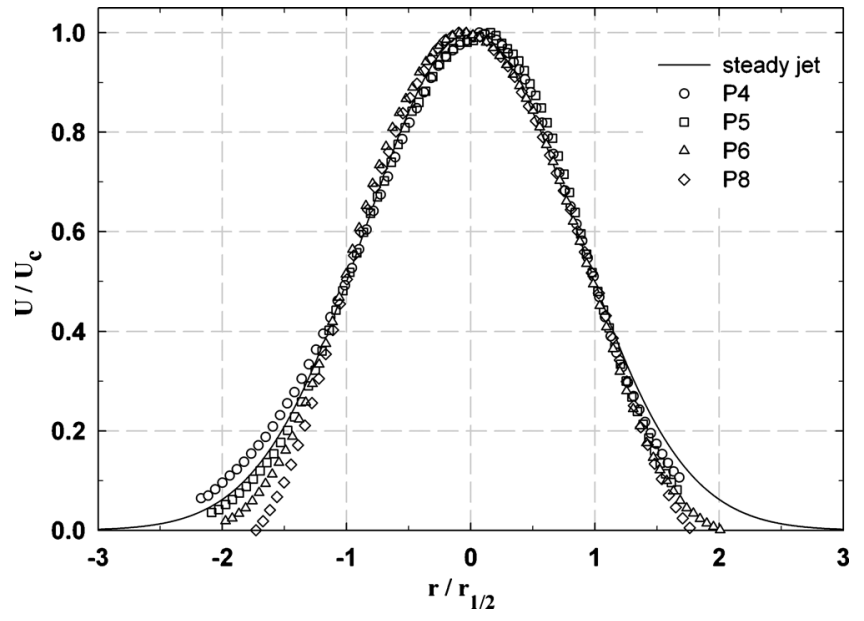

FIG. 5. Axial velocity profile through the puff center. The steady jet (solid line) is represented by the best-fit Gaussian.

width $r_{1 / 2}=\delta_{1 / 2} / 2$. The puff half-widths along with those of the steady jet are shown in Fig. 4. As expected, the jet halfwidth increases linearly with the axial distance. The puffs have half-widths greater than the jet at the same $z$ location and the puff widths decrease as $P$ increases. The $P=4$ puff has the largest relative half-width, $\sim 20 \%$ greater than the jet width at the same location.

The mean velocity profile for each puff was normalized by the velocity at the puff center $U_{c}$ and plotted in similarity coordinates $\left(U / U_{c}\right.$ versus $\left.r / r_{1 / 2}\right)$ in Fig. 5. In the range of $-1 \leq r / r_{1 / 2} \leq 1$, the axial velocity profiles at puff center nearly collapse onto the steady jet profile. Beyond the puff half-radius, however, there are some variations. The overall width of the profiles decrease as $P$ increases, with the $P=4$ puff having the largest width. This is consistent with the half-width data in Fig. 4. The profiles in Fig. 5 were fitted by a Gaussian as follows with $k$ and $r_{o}$ as the fitting parameters:

$$
U=U_{c} \exp \left\{-k\left[\left(r-r_{o}\right) / r_{1 / 2}\right]^{2}\right\} .
$$

The $r_{o}$ parameter was used since the average puff center may be slightly off-center. Only the $P=6$ case was perceptibly off-center with $r_{o}=0.2 d$. The peak value of $U / U_{c}$ for puffs may occur off-center due to the jitter in the radial position of the puff and the averaging of such images. The fitting parameter was found to range from $k=0.69$ for the $P=4$ puff to 0.84 for the $P=8$ puff. The steady jet value was $k=0.695$. In the experiments of Bremhorst and Hollis, ${ }^{3}$ a value of $k=0.69$ was found for a $P=3.6$ puff at $\mathrm{Re}=6$ $\times 10^{4}$, equal to our $P=4$ value. Hence, the mean velocity profiles at the puff center can be characterized by a Gaussian distribution with $k$ values comparable to the steady jet in the similarity coordinates.

\section{Fluctuating velocity}

Contours of axial velocity fluctuation $u_{\mathrm{rms}}^{\prime}$ normalized by the maximum velocity at the puff center are shown in Fig. 6 for the $P=4,6$, and 8 puffs, and the steady jet. For the steady jet, $u_{\mathrm{rms}}^{\prime}$ was normalized by the centerline velocity at the same axial location. These contour plots clearly indicate that the peak velocity fluctuations for the puffs occur above the 

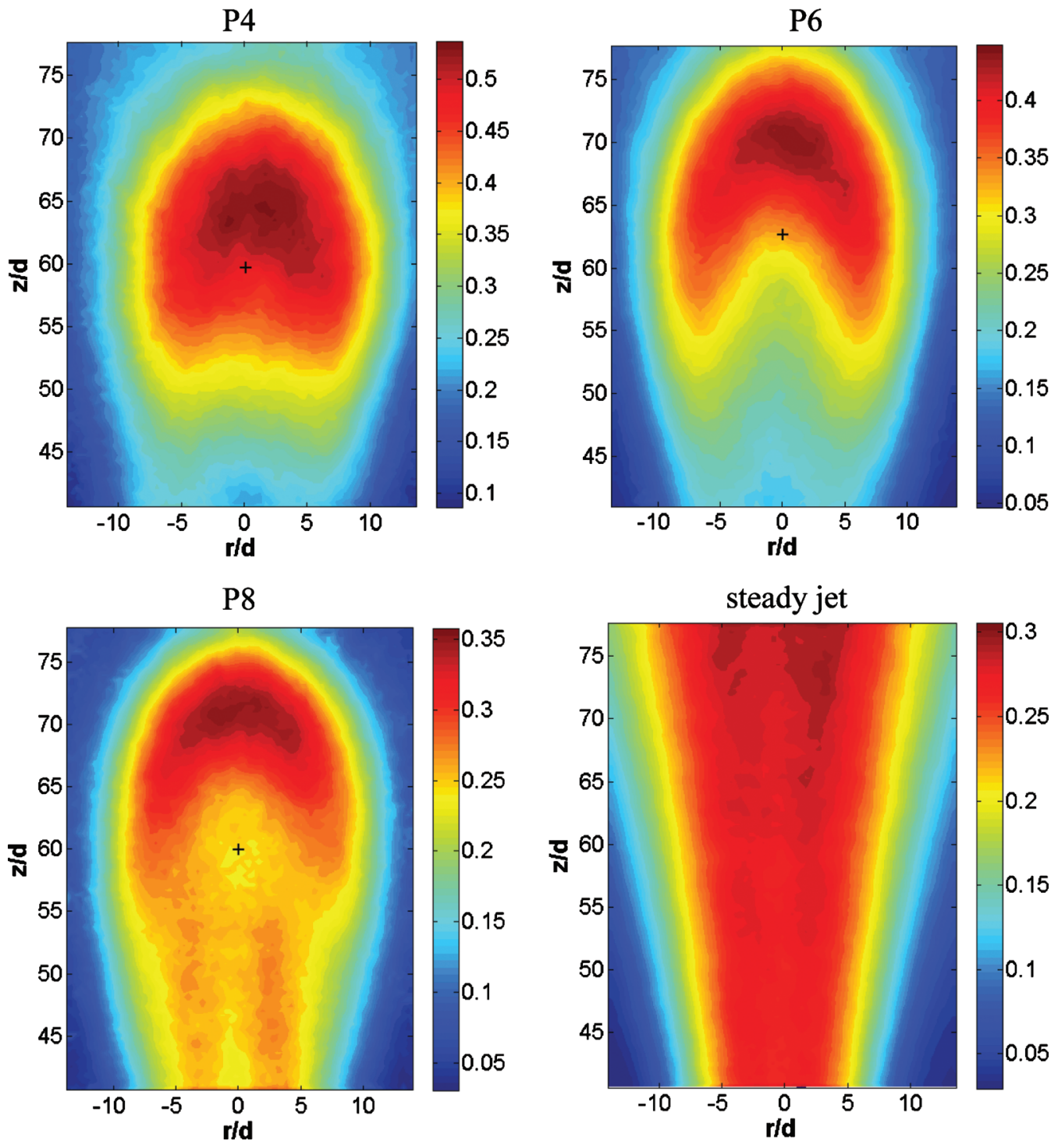

FIG. 6. (Color) Scaled axial fluctuation velocity $u_{\mathrm{rms}}^{\prime} / U_{c}$ contours for the puffs and the steady jet. The plus sign denotes the puff center.

puff center and that the contours are not symmetric top and bottom. The high value contours for the puffs form a chevron shape. By moving from the puff center to the trailing edge, the $u_{\mathrm{rms}}^{\prime}$ values decrease monotonically while moving to the puff leading edge, $u_{\mathrm{rms}}^{\prime}$ first increases before decreasing to the zero. The chevron profile becomes more prominent with increasing $P$. The lower value contours for the $P=8$ resembles the perimeter of a starting jet. The steady jet contours in contrast display an expanding conical flow regime.

Radial profiles of the normalized axial velocity fluctuation $u_{\mathrm{rms}}^{\prime} / U_{c}$ along the puff center are plotted against $r / r_{1 / 2}$ in Fig. 7. For the steady jet, the profile at $60 d$ is shown in Fig. 7. The steady jet profile has an off-center peak value of $0.28 U_{c}$, the same value found by Hussein et al. ${ }^{17}$ The $u_{\mathrm{rms}}^{\prime}$ profiles for the puffs are nearly symmetric about the centerline and also have an off-center peak. The off-center peaks correspond to the chevron shape of the high value contours in Fig. 6 . The $P=4$ puff has the highest relative $u_{\mathrm{rms}}^{\prime}$ with a peak value of $0.51 U_{c}$ (average of the left and right side) among the cases examined. The maximum magnitude of $u_{\mathrm{rms}}^{\prime} / U_{c}$ for the $P=4$ puff is about twice the steady jet value.
The data in Fig. 7 indicate that the $u_{\mathrm{rms}}^{\prime} / U_{c}$ profiles get closer to the steady jet profile as $P$ increases from 4 to 8 . In fact, the values near the centerline for the $P=8$ puff and the steady jet are quite close. Even disregarding the relative amplitudes,

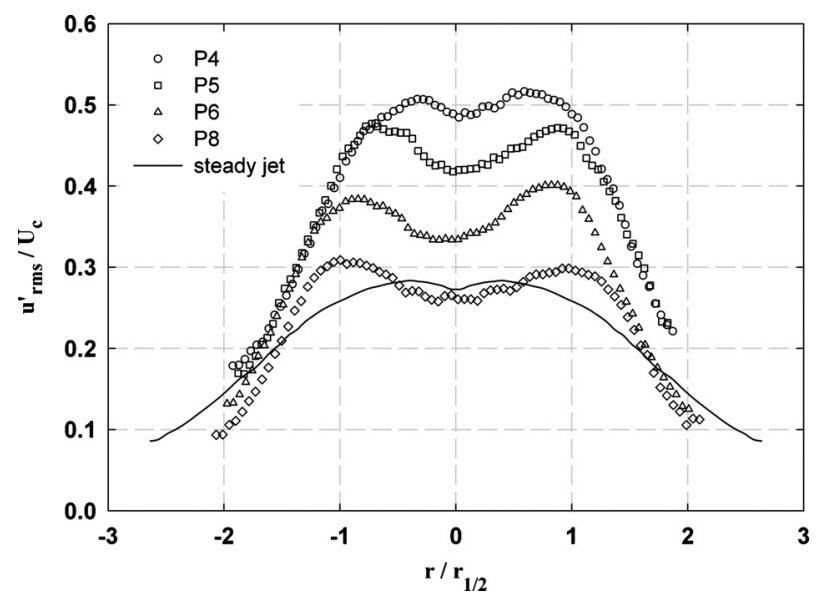

FIG. 7. Axial fluctuation velocity profiles in similarity coordinates. 

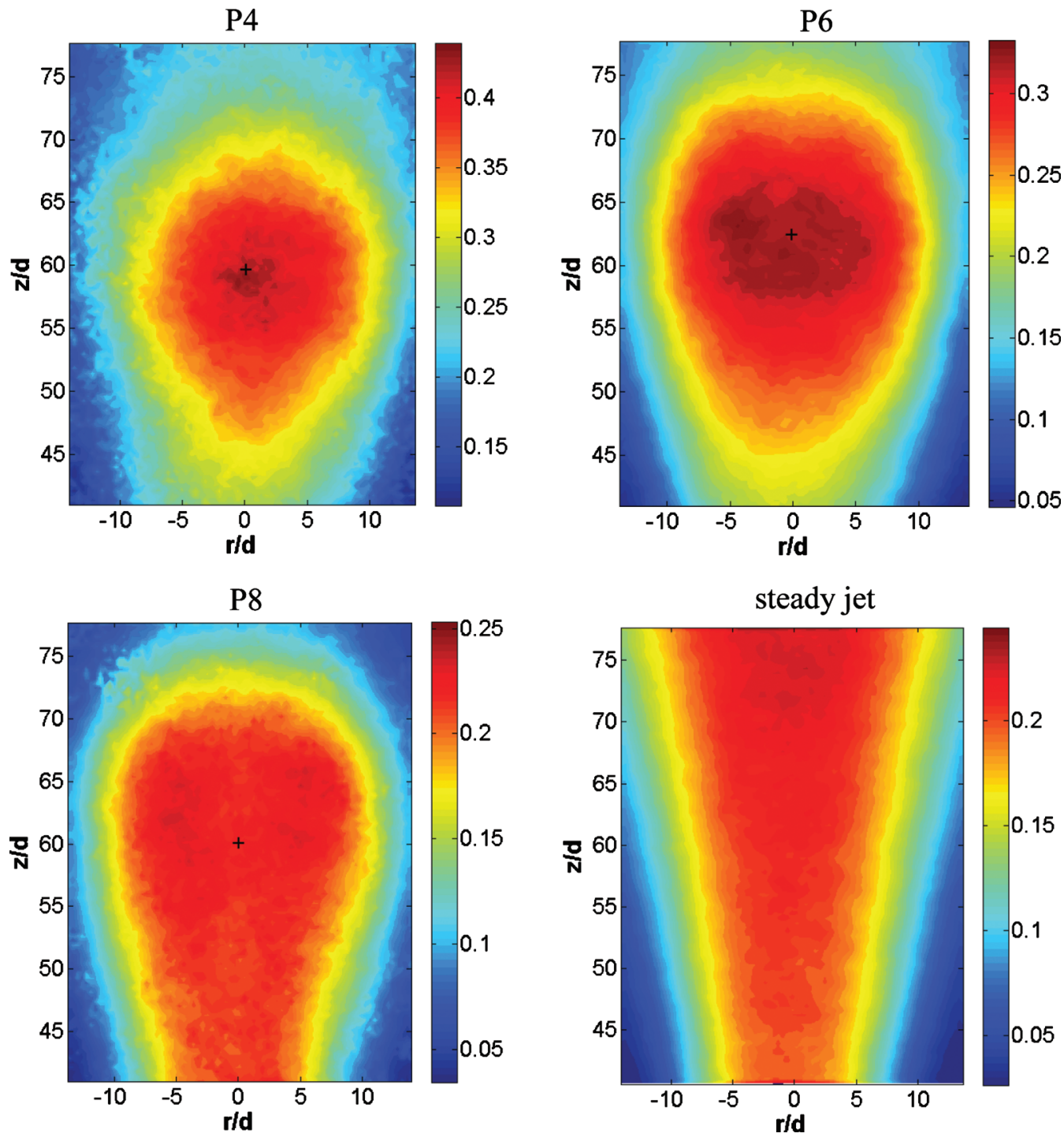

FIG. 8. (Color) Scaled radial fluctuation velocity $v_{\mathrm{rms}}^{\prime} / U_{c}$ contours for the puffs and the steady jet. The plus sign denotes the location of puff center.

the $u_{\mathrm{rms}}^{\prime}$ profiles in Fig. 7 cannot be forced to collapse together as the location of the peak varies for the four puff cases.

Contours of radial velocity fluctuation $v_{\text {rms }}^{\prime}$ normalized by the maximum velocity at the puff center are shown in Fig. 8 for the $P=4,6$, and 8 puffs, and the steady jet. For the steady jet, $v_{\text {rms }}^{\prime}$ was normalized by the centerline velocity at the each axial location. These contour plots reveal that the maximum value of $v_{\text {rms }}^{\prime}$ occurs near the puff center in contrast to the $u_{\mathrm{rms}}^{\prime}$ peaks that were located above the puff center. The higher level $v_{\mathrm{rms}}^{\prime}$ contours have rounded shapes for the $P=4$ and 6 cases, and the $P=4$ puff has the highest value of $v_{\mathrm{rms}}^{\prime}$. The lower level contours representing the puff boundary elongate axially as $P$ increases and for the $P=8$ puff resemble the head of a starting jet. Indeed, the lower level $v_{\mathrm{rms}}^{\prime}$ and $u_{\mathrm{rms}}^{\prime}$ contours for the $P=8$ are quite similar. In contrast to the puffs, the steady jet contours show an expanding conical flow regime.

The profiles of radial velocity fluctuation along the puff center were extracted from the data in Fig. 8 and are plotted in similarity coordinates $\left(v_{\mathrm{rms}}^{\prime} / U_{c}\right.$ versus $\left.r / r_{1 / 2}\right)$ in Fig. 9.
The $v_{\text {rms }}^{\prime}$ profiles are almost symmetric about the centerline and the peak is near the centerline. As with the $u_{\mathrm{rms}}^{\prime}$ profiles, the $P=4$ puff has the highest relative $v_{\text {rms }}^{\prime}$ with a peak value of $0.43 U_{c}$ among the four puff cases considered. The maxi-

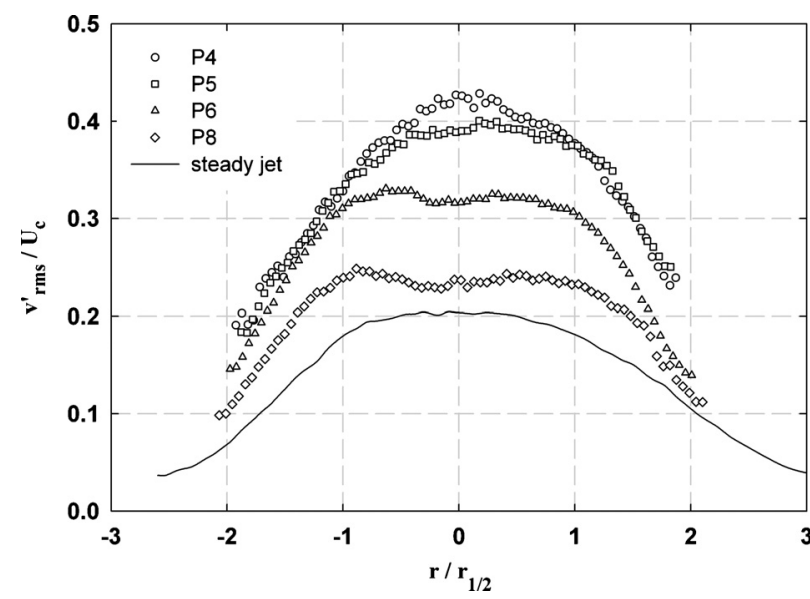

FIG. 9. Radial fluctuation velocity profiles in similarity coordinates. 
P4

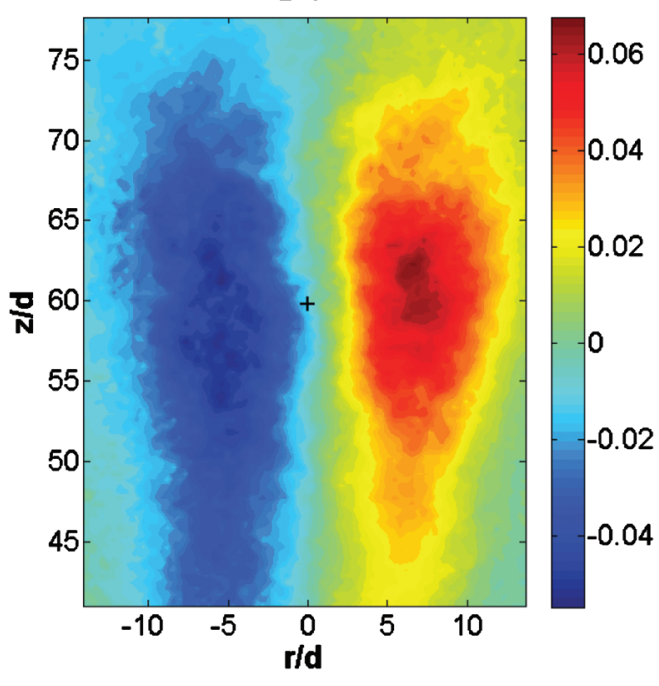

P8

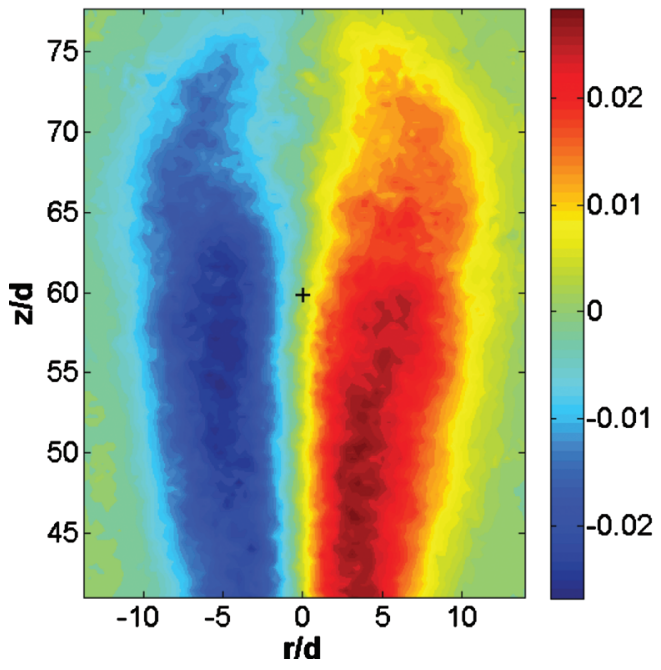

P6

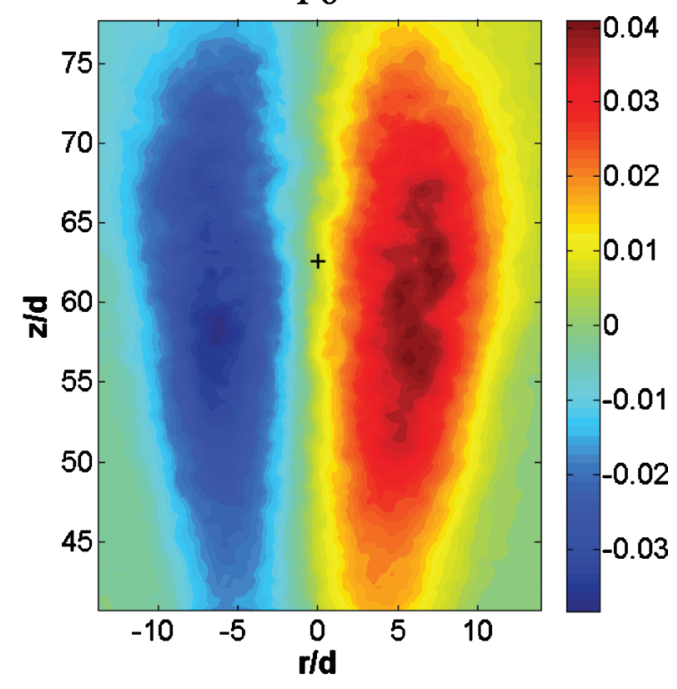

steady jet

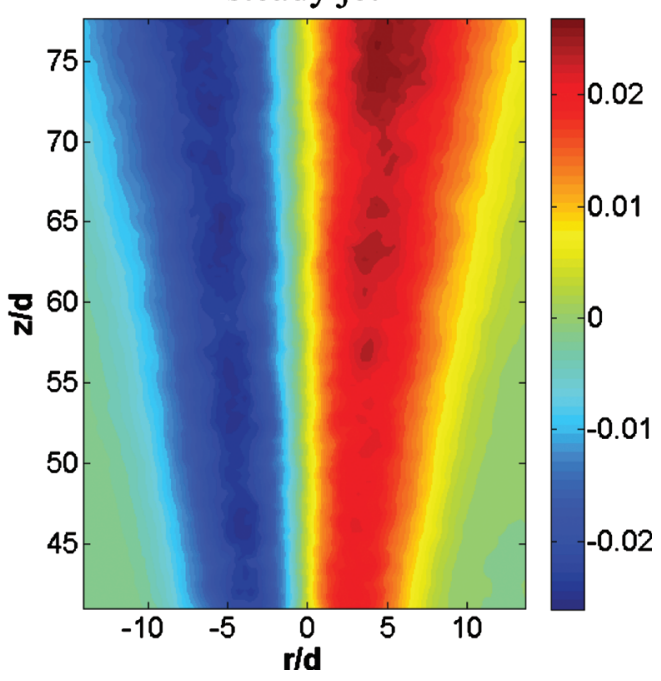

FIG. 10. (Color) Normalized turbulent shear stress contours for the puffs and the steady jet. The plus sign denotes the location of puff center.

mum magnitude of $v_{\mathrm{rms}}^{\prime} / U_{c}$ for the $P=4$ puff is over twice the steady jet value. As $P$ increases from 4 to 8 , the peak of $v_{\text {rms }}^{\prime}$ profiles decreases and the normalized profiles get closer to the steady jet profile. The steady jet profile is closest to the $P=8$ puff profile. All four puff profiles have higher values of $v_{\mathrm{rms}}^{\prime} / U_{c}$ than the steady jet.

\section{Turbulent shear stress}

The turbulent shear stress term $\left\langle u^{\prime} v^{\prime}\right\rangle$ was computed from the phase-locked data sets to assess whether this turbulent stress transport component depends on $P$ to the same degree as the velocity fluctuations. The shear stress was normalized by the square of the maximum velocity at the puff center and the contour plots for the $P=4,6$, and 8 puffs, and the steady jet are shown in Fig. 10. For the steady jet, $\left\langle u^{\prime} v^{\prime}\right\rangle$ was normalized by the square of the centerline velocity at each axial location. The turbulent shear stress is antisymmetric about the centerline as expected and the contours show an elongated tail even for the $P=4$ puff. The maximum shear stress location is off the centerline and about at the same level as the puff center. The sign of the turbulent shear stress indicates that the direction of the turbulent stress transport is away from the puff center. The data in Fig. 10 also indicate that the shear stress contours, especially at the higher values, get stretched out axially as $P$ increases. Similar to the velocity fluctuations, the steady jet contours exhibit the conical expansion of the flow.

Radial profiles of the dimensionless shear stress $\left\langle u^{\prime} v^{\prime}\right\rangle / U_{c}^{2}$ along the puff center were extracted from the data and are plotted against $r / r_{1 / 2}$ in Fig. 11. The steady jet shear stress profile is self-similar in the studied range. The turbulent shear stress profiles for the puffs follow a trend similar to the steady jet except with different peak magnitudes. The shear stress is positive where the mean shear $(\partial U / \partial r)$ is negative; the sign of the shear stress agrees with the net transport of high momentum away from the centerline. The peak value decreases as $P$ increases and eventually becomes nearly the same as the steady jet for the $P=8$ puff. The $P=4$ puff has the highest value of $\left\langle u^{\prime} v^{\prime}\right\rangle / U_{c}^{2}$ with a maximum that is over twice the steady jet value. The radial loca- 


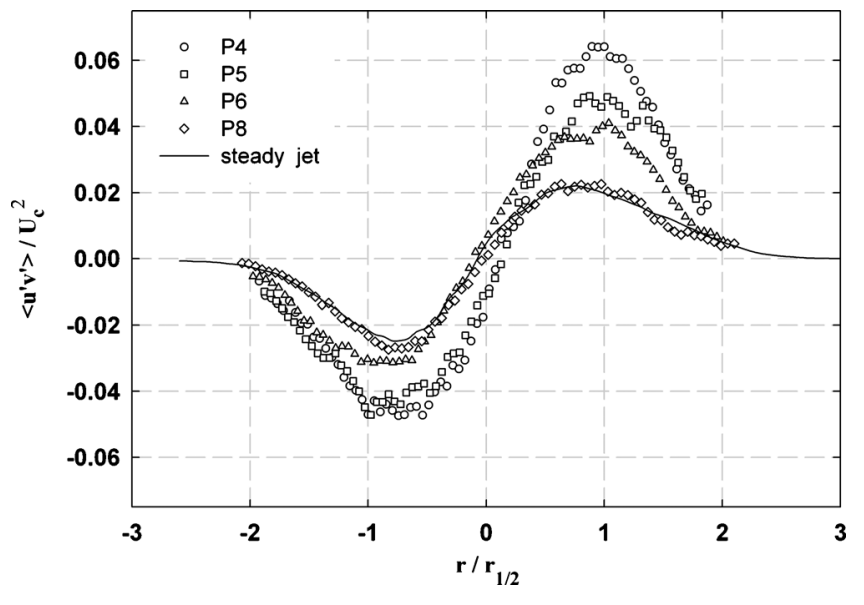

FIG. 11. Turbulent shear stress profiles in similarity coordinates.

tion of the peak shear stress in terms of the half-radius is comparable among the cases considered. The maximum values of the axial and radial velocity fluctuations as well as the turbulent shear stress for the puffs and the steady jet are listed in Table I. The values are the average of the left and right sides of the data.

\section{E. Vorticity}

The azimuthal vorticity field was computed from the measured velocity data to explore the vorticity distribution within puffs. The mean azimuthal vorticity was scaled by the nozzle diameter and the velocity at the puff center $\omega d / U_{c}$ and is plotted in Fig. 12 for the $P=4,6$, and 8 puffs in addition to the steady jet. For the steady jet, the centerline velocity at the corresponding axial location was used for scaling. The plots in Fig. 12 reveal that vorticity is present throughout the puff volume, except on the centerline. The data also illustrate the presence of an elongated ringlike structure. In contrast to vortex rings with circular crosssection, the higher value contours for the puffs in Fig. 12 have an elliptical cross-section with major axis in the flow direction. The elliptical cross-sections of these contours have aspect ratios of 2-3. Another major difference between the puffs and their vortex ring counterparts is the large "vortex" diameter (the distance between the left and right peak vorticity locations) of the puffs. Assuming the virtual origin of the puffs to be at the nozzle, the spreading rate of the puffs in this study is an order of magnitude larger than those of the turbulent vortex rings reported by Maxworthy. ${ }^{21}$

The magnitude of the scaled vorticity is comparable for the various puffs and does not change much as $P$ increases. This indicates that the scaled mean vorticity is significantly less dependent on $P$ than the turbulent shear stress or velocity fluctuations. The contour plots in Fig. 12 also show that the ringlike structure becomes further elongated as $P$ increases. The vorticity distribution for the $P=8$ puff is different than the other puffs as there is significant vorticity in the tail region of the puffs. Vorticity decreases toward the trailing edge of the lower $P$ puffs. This is indicative of the $P=8$ puff vortical structure being similar to a starting jet with a ringlike head and a jetlike tail. Given that the $P=8$
TABLE I. Maximum values of scaled axial and radial velocity fluctuations and turbulent shear stress.

\begin{tabular}{lccc}
\hline \hline$P$ & $\left(u_{\mathrm{rms}}^{\prime} / U_{c}\right)_{\max }$ & $\left(v_{\mathrm{rms}}^{\prime} / U_{c}\right)_{\max }$ & $\left(\left\langle u^{\prime} v^{\prime}\right\rangle / U_{c}^{2}\right)_{\max }$ \\
\hline 4 & 0.51 & 0.43 & 0.056 \\
5 & 0.47 & 0.40 & 0.048 \\
6 & 0.39 & 0.33 & 0.036 \\
8 & 0.30 & 0.22 & 0.025 \\
Steady jet & 0.28 & 0.20 & 0.023 \\
\hline \hline
\end{tabular}

puff structure is close to the head of a starting jet and the $P=4$ appears as fully formed puff, the changes in the vorticity distribution going from $P=8$ to 4 indicate approximately the spatial development of the puff vorticity field. The steady jet vorticity field shows the conical expansion of the flow and resembles the shear stress distribution in Fig. 10.

\section{F. Entrainment}

Entrainment may be qualitatively defined as the engulfment of irrotational fluid into the turbulent region. The entrainment rate controls the mixing rate of the source flow with the ambient fluid in most turbulent flows. Entrainment in equilibrium turbulent flows is related to the Reynolds stresses. ${ }^{3}$ Results of Sec. III D have shown that puffs have significantly greater Reynolds stress values than the steady jet at the same Reynolds number. Therefore, entrainment by a puff is expected to be different than that of the steady jet.

The mass flow rate in steady jets increases continuously by entrainment and one method for determining the entrainment rate of steady jets involves computing the rate of increase of mass flux in the axial direction by integrating the mean velocity across the jet cross-section. As the mean velocity profile is Gaussian in the self-similar regime, the centerline velocity $U_{c}$, half-radius $r_{1 / 2}$, and the parameter $k$ from Eq. (4) are sufficient to compute the jet mass flux $\dot{m}=\rho U_{c} \pi r_{1 / 2}^{2} / k$. Since $U_{c}$ decreases inversely with $z$ and $r_{1 / 2}$ increases linearly with $z$ for a steady jet, the jet mass flux of the jet should increase linearly with $z \cdot{ }^{22,23}$ The computed data for the steady jet was linear, indicating a constant entrainment rate for the axial range studied. The normalized jet entrainment rate $d\left(\dot{m} / \dot{m}_{o}\right) / d(z / d)$ in the range examined was 0.31 , which is comparable to the accepted value of 0.32 . The same method cannot be used to determine the puff entrainment rate as the puff flow is unsteady. An alternative method is pursued below.

The increasing flow rate in the homogeneous steady jet is accommodated by the radial inflow of the ambient fluid. Indeed, the entrainment rate $d \dot{m} / d z$ is equal to $-2 \pi r \rho V$ at large $r$. To investigate the entrainment in puffs, the parameter $2 \pi r V$ was extracted from the (right side) velocity data at the puff center and plotted against $r / r_{1 / 2}$ in Fig. 13. For the steady jet, the profile at $60 d$ was used. Negative values of $2 \pi r V$ indicate radial inflow and entrainment while positive values reflect radial outflow or expansion. The data reveal that no inflow or entrainment takes place at the center of the four puff cases considered and only radial expansion is 

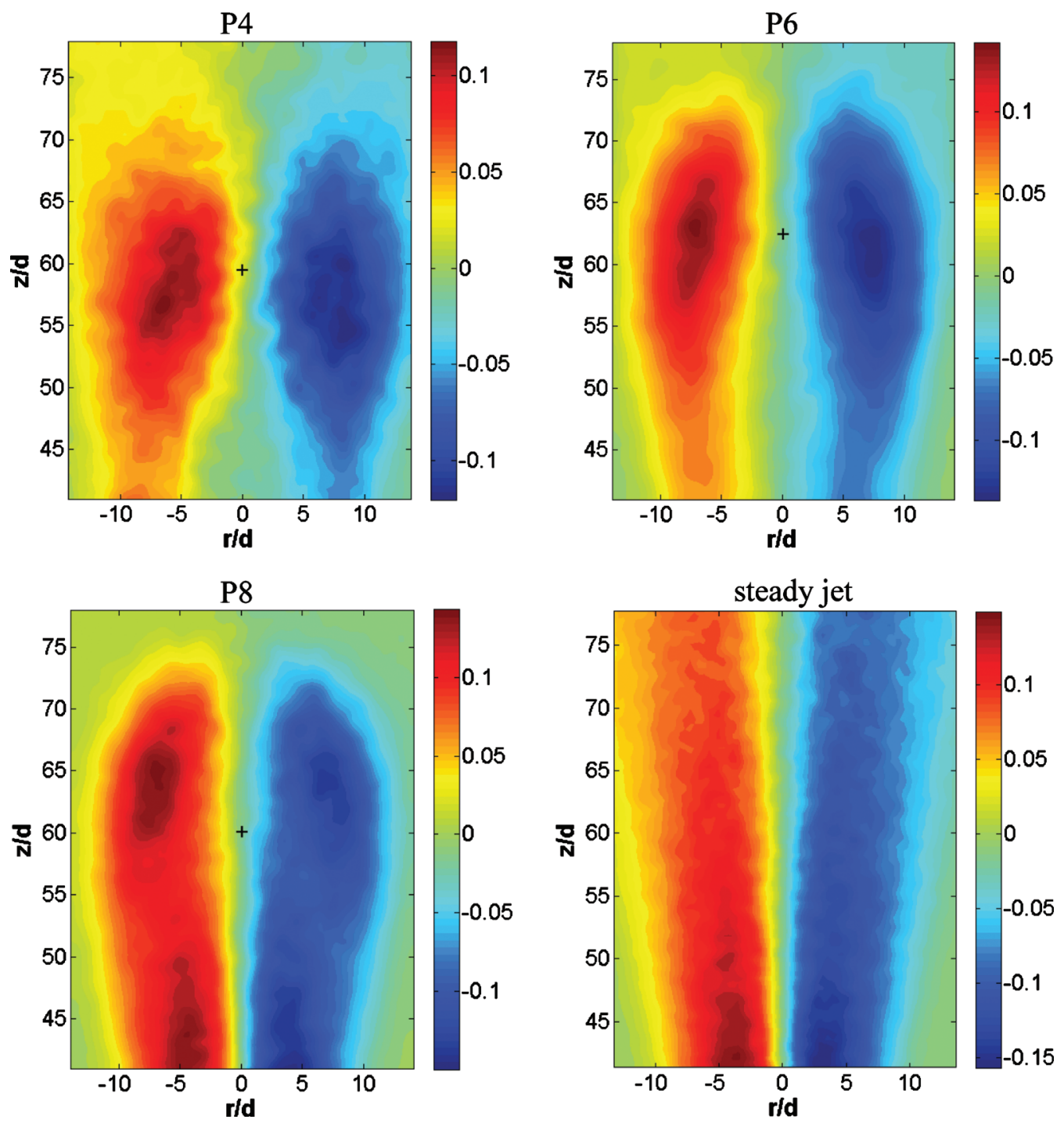

FIG. 12. (Color) Normalized vorticity contours $\left(\omega d / U_{c}\right)$ for the puffs and the steady jet. The plus sign denote the location of puff center.

present. On the other hand, the steady jet shows radial outflow for $r \leq 1.4 r_{1 / 2}$ and inflow thereafter. This is consistent with the notion of the steady jet entraining from its sides. The data in Fig. 13 also illustrate that unlike the steady jet, the centerline velocity and half-radius at the puff center cannot be used to assess the entrainment rate.

To further explore the entrainment pattern in turbulent puffs, the radial profiles of $2 \pi r V$ at seven axial stations for the $P=4$ and 8 puffs were computed and plotted in Fig. 14 . The seven stations consist of three upstream of the puff center at $45 d, 50 d$, and $55 d$, three downstream of the puff center at $65 d, 70 d$, and $75 d$, as well as the puff center. For the $P=4$ puff, the data in Fig. 14(a) indicate that radial inflow and entrainment takes place upstream the puff center, while radial expansion occurs at and above the puff center. A similar pattern of entrainment and radial expansion is also exhibited by the $P=8$ puff data in Fig. 14(b), as well as by the two other cases at $P=5$ and 6 . The magnitudes of $2 \pi r V$ at the larger radial positions, which are indicative of the entrainment rate, reveal that the entrainment rate below the puff center is greater than the radial expansion for the $P=4$ puff. On the other hand, the radial expansion appears to be larger than the entrainment rate for the $P=8$ puff. Comparing the magnitudes of inflow below the puff center with the radial expansion above the puff center for the four cases suggests that the relative entrainment is greater for the lower $P$ puffs.

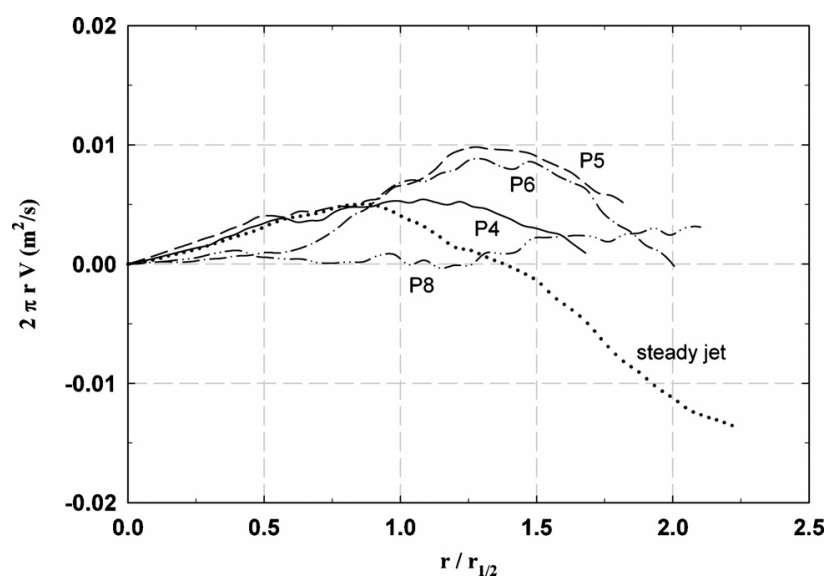

FIG. 13. Profiles of $2 \pi r V$ at puff center for the puffs and the steady jet at $60 d$. 

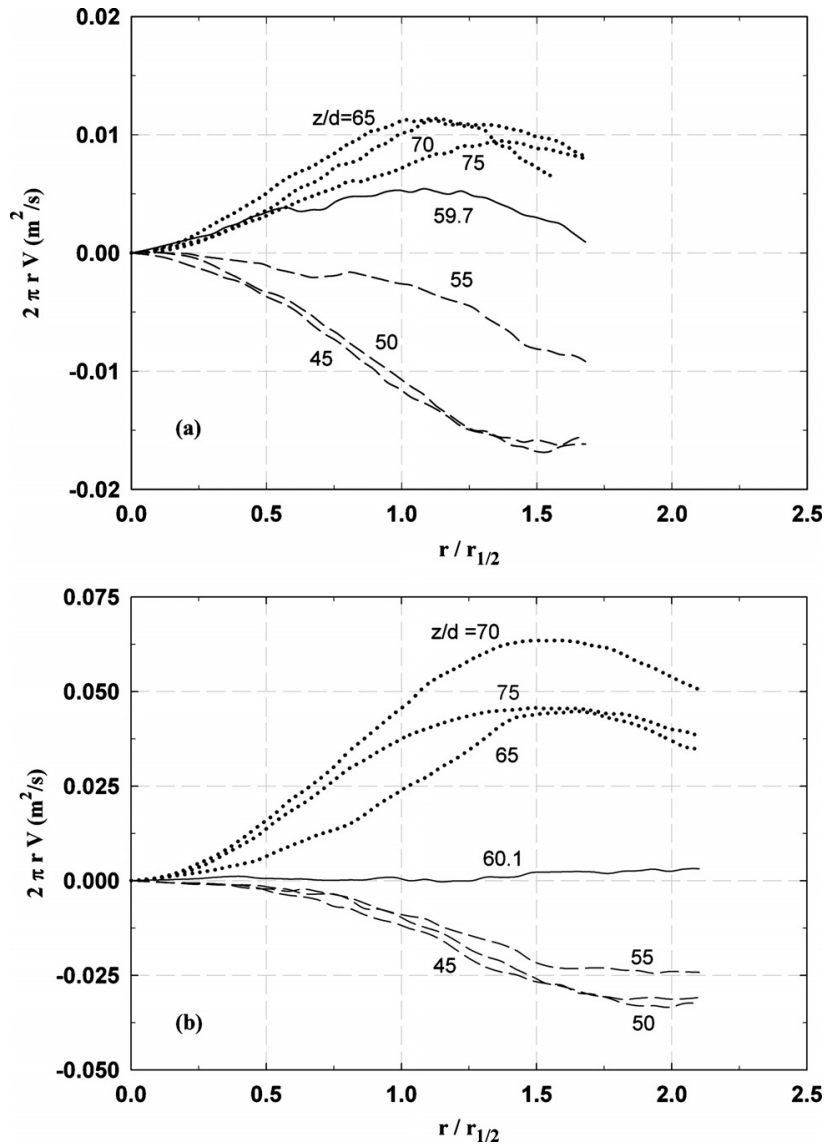

FIG. 14. Profiles of $2 \pi r V$ for the puffs with (a) $P=4$ and (b) $P=8$. The solid lines represent the puff center, the dashed lines below, and the dotted lines above the puff center.

To visualize the entrainment pattern, the streamlines in a reference frame moving with the puffs was computed. This was done by subtracting the puff celerity from the mean velocity field. The resulting streamlines are presented in Fig.
15 for the $P=4$ and 8 puffs. The streamline patterns are very sensitive to the puff celerity used, as noted likewise in Ref. 10 , and any asymmetry in the measured velocity field. The streamlines for the $P=4$ puff reveal a prolate ellipsoid, slightly tilted to one side, whereas the $P=8$ puff streamlines appear similar to a starting jet. The streamlines patterns are consistent with the mean velocity contours in Fig. 3. The $P=4$ puff pattern shows entrainment below the puff center on the right side and a closed recirculation volume on the left side. The left/right side difference is attributed to a slight asymmetry of the measured data. The streamlines for the $P=8$ case appear quite distinct from those of the $P=4$ puff in that the enclosed volume extends from the upper stagnation point to the bottom of the measurement plane. Flow clearly moves up into the puff from the bottom and forms the recirculation pattern inside the puff boundary. These streamline patterns indicate that the entrainment takes place on the bottom portion of the puff in corroboration of the radial velocity profiles in Fig. 14.

Based on the findings in this section, a simplified schematic of the entrainment pattern is presented in Fig. 16, where the majority of entrainment into the puff occurs from below the puff center while the puff cap pushes out into the surrounding fluid. Of course, the details of the radial inflow/ outflow above and below the puff center depend on $P$.

\section{G. Burning puffs}

As noted in Sec. I, fully modulated, reacting jets of hydrocarbon fuels produce flames that are shorter than that of the steady jet. The shortest flame lengths have been observed for conditions resulting in individual, compact flame structures. ${ }^{5,6,24,25}$ These compact flames are analogous to the nonreacting, homogeneous, turbulent puffs considered in this study. The flame length of a $P=4$ puff could be as small as a quarter of a steady jet flame.
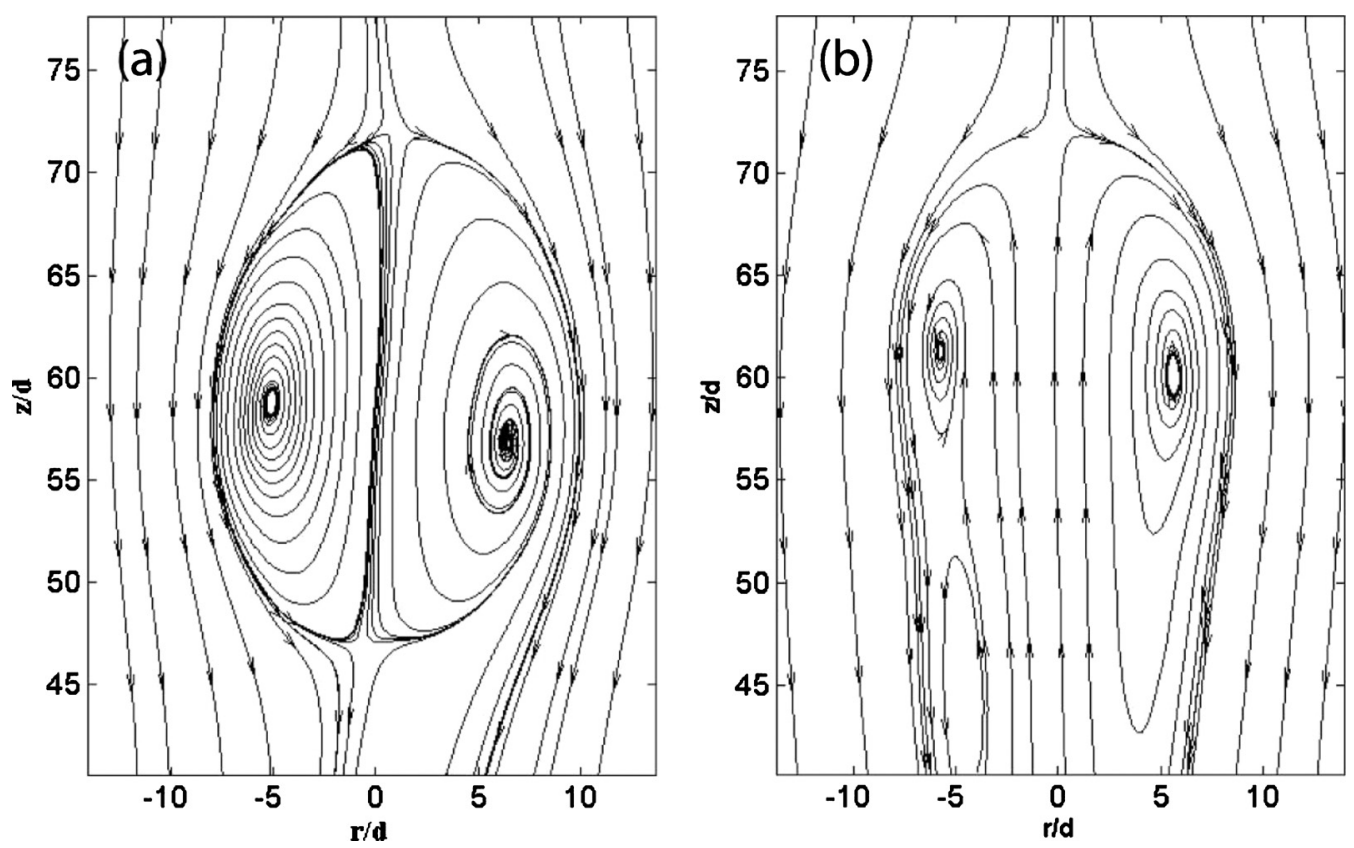

FIG. 15. Streamlines in a reference frame moving with the puff (a) $P=4$ and (b) $P=8$. 


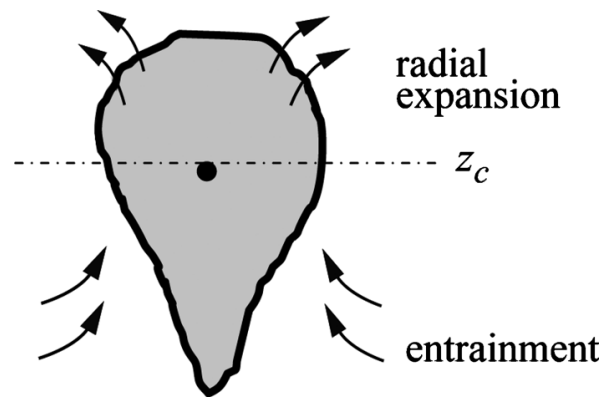

FIG. 16. Schematic of the puff entrainment pattern.

The experiments of Hermanson et al. ${ }^{24}$ utilized a setup very similar to the present one. Unheated ethylene was injected through a $2 \mathrm{~mm}$ diameter nozzle into a coflow chamber and ignited using a heated element near the nozzle; the length of visible flame was recorded. The nozzle Reynolds number was 5000, the same as the present experiments. The average flame length of burning puffs from this experiment is listed in Table II. The puff flame length increased linearly with $P$, up to $P \approx 11 .^{24}$ The image of a burning puff in Fig. 2 of Ref. 24 shows that the tail of a $P=8$ puff burns out first compared with the cap of the puff. This is consistent with the pattern of entrainment in Fig. 16 where entrainment takes place on the lower side of the puff. Furthermore, the data in Table II reveal that the changes in the flame length correlate with the relative increase in the entrainment by the lower $P$ puffs and the variation in the entrainment rate is at least partially responsible for the observed shorter flame lengths. Hence, the results for the homogenous puffs found here appear to be applicable, at least qualitatively, to the case of burning puffs and fully modulated jets. It should be noted that heat release and the associated buoyancy play a role in the puff flame lengths and these effects are not addressed in this study.

\section{H. Comparison with past studies}

To examine the findings of current study in light of past investigations, two specific cases are considered. The most relevant case is the velocity magnitude measurements of Kovasznay et al.; their contour plot is shown in Fig. 17. As mentioned in Sec. I, the flow field in the near field of a $P \approx 2.5$ puff was measured using a hot-wire anemometer. The contours in Fig. 17 may be contrasted against with the $P=4$ puff data in Fig. 3. The velocity distribution in the center of $P=4$ puff is generally comparable with the contours having values greater than 0.5 in Fig. 17. On the other hand, the lower value contours in Fig. 17 extend upstream to

TABLE II. Flame length of burning puffs.

\begin{tabular}{cc}
\hline \hline$P$ & Flame length \\
\hline 4 & $59 d$ \\
5 & $85 d$ \\
6 & $110 d$ \\
8 & $160 d$ \\
\hline
\end{tabular}

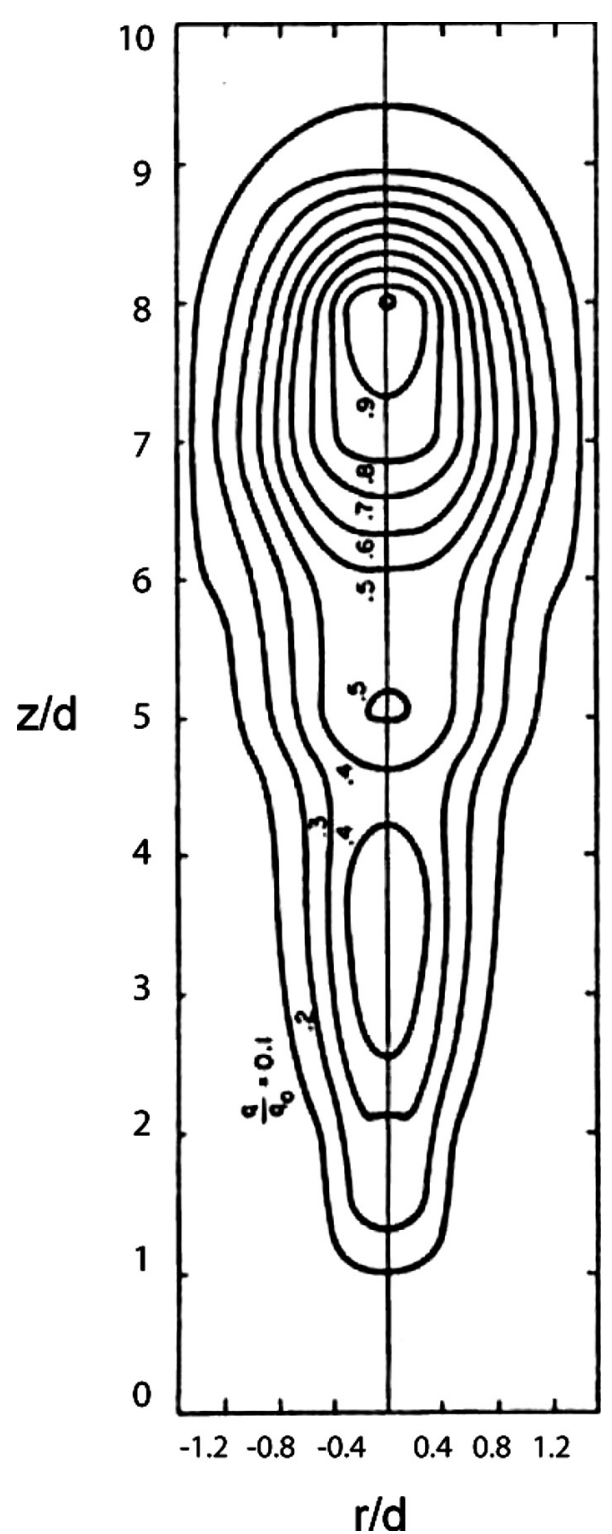

FIG. 17. Velocity magnitude contours from Kovasznay et al. (Ref. 8).

the vicinity of the nozzle. This was not the case for the $P=4$ puff in the current experiments. The difference stems from the proximity of the puff to the nozzle; the last parcels of injected fluid did not have time to get entrained into the rest of the puff. It is thought that had the same measurements been carried out further away from the puff source in Ref. 8, the velocity contours would be much more similar to those of the $P=4$ puff.

The other case considered was the turbulent vortex ring of Glezer and Coles. ${ }^{10}$ The flow field of a vortex ring with an equivalent $P=1.5$ was surveyed using the Laser-Doppler technique. It is worth noting that only for $P<1.6$ individual vortex rings can be created. ${ }^{13}$ The data for this vortex ring are particularly relevant as both the vorticity and fluctuating velocity contours are reported in Ref. 10. Contours of the mean azimuthal vorticity and axial normal Reynolds stress $\left\langle u^{\prime} u^{\prime}\right\rangle$ from Ref. 10 are shown in Fig. 18. The coordinates have been scaled to be comparable to the axial range considered in the present study; the original data were in similarity 

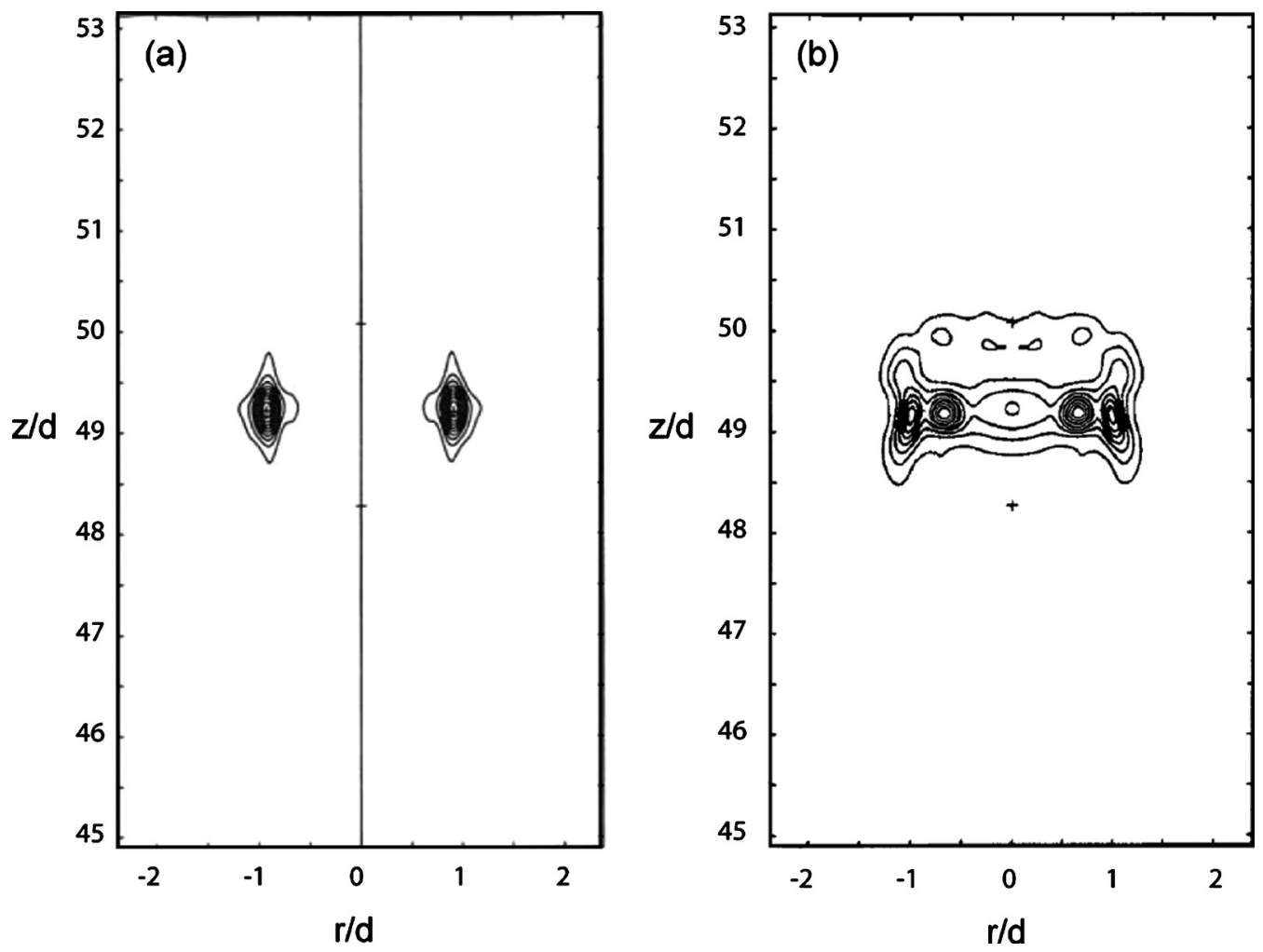

FIG. 18. (a) Mean vorticity and (b) axial normal Reynolds stress reproduced from Ref. 10.

coordinates. The vorticity contours in Fig. 18(a) may be compared with the data in Fig. 12. The vorticity in the $P=4$ puff is spread over a much larger area in contrast to the compact contours of the vortex ring in Fig. 18(a). However, the cross-section of the vortex core and the $P=4$ puff vorticity contours appear similar albeit at different sizes.

The scaled axial normal Reynolds stress contours in Fig. 18(b) may be examined against the fluctuating axial velocity data for the $P=4$ puff in Fig. 6 . The vortex ring data indicate that the normal Reynolds stress is concentrated around the vortex core and the ring centerline has minimal normal stress. On the other hand, the scaled $u_{\mathrm{rms}}^{\prime}$ contours in Fig. 6 enclose a large volume encompassing the majority of the puff and the higher valued contours have chevron-shaped outlines. Thus, the structure of the puff flow field differs quite a bit from the turbulent vortex ring. The differences are attributed to vorticity being concentrated within the vortex core in contrast to the widespread presence of vorticity within the puff.

\section{CONCLUSIONS}

The velocity field of nonreacting, isolated, turbulent puffs was measured by the PIV technique in a diametrical plane. The puffs considered in this study began as starting jets and, upon termination of the flow from the nozzle, evolve into large scale turbulent structures with vorticity present throughout their volume. For the larger $P$ puffs, a larger volume is injected and a greater axial distance is needed to get all the injected fluid entrained into the puff. This is the reason for the different flow structures observed within the $40 d-75 d$ range in this study. The significantly larger scaled velocity fluctuations and turbulent shear stress are a result of the rapid mixing and decay of the mean puff velocities $\left(\sim z^{-3}\right)$. As puffs are nonstationary flows, the turbulent fluctuations lag behind the mean flow field. Thus, turbulent fluctuations scaled with the mean centerline velocity become much larger than those in a stationary flow such as the steady jet. The entrainment pattern of puffs is different than that of other turbulent flows, such as the jet, as only ambient fluid is entrained from the bottom of the puff. The principal findings of this work over the axial range examined can be summarized as follows:

- The averaged velocity contours showed that the puffs evolve from a prolate geometry to one with a "tail" as the injection volume increases. The axial velocity profile at the puff center follows a Gaussian distribution. The puff center half-widths decrease with increasing $P$ and get closer to the steady jet value. The mean and fluctuating velocity as well as turbulent shear stress profiles also got closer to the steady jet as $P$ increases.

- The axial and radial velocity fluctuations corresponding to the location of puff center were nearly symmetric about the centerline. The peak values of axial and radial velocity fluctuations among the puffs were observed for the $P=4$ puff. The maximum magnitude of scaled axial and radial velocity fluctuations for the $P$ $=4$ puff was about twice the steady jet value.

- The turbulent shear stress profiles at the puff center were observed to follow a trend similar to the steady jet's but at different magnitudes, especially for the 
lowest $P$ puff. The maximum level of the scaled turbulent shear stress for the $P=4$ puff was about 2.4 times the steady jet value.

- The vorticity field showed the presence of vorticity throughout the puff volume, unlike that in turbulent vortex rings. The scaled vorticity magnitude did not change appreciably for the four puff cases in contrast to the fluctuation velocities values.

- Entrainment takes place below the puff center and only radial expansion is present at or above the puff center. Comparing the inflow below with the expansion above the puff center reveals that the entrainment is greater for the lower $P$ puffs.

\section{ACKNOWLEDGMENTS}

This work was supported by the NASA Microgravity Research Division under Cooperative Agreement No. NNC04GA61G. The experiments were carried out at Worcester Polytechnic Institute, Worcester, MA. Useful discussions with J. C. Hermanson, U. Hegde, and D. Stocker are greatly appreciated.

${ }^{1}$ Y. M. Tang, G. Waldherr, J. I. Jagoda, and B. T. Zinn, "Heat release timing in a nonpremixed Helmholtz pulse combustor," Combust. Flame 100, 251 (1995).

${ }^{2}$ S. C. Crow and F. H. Champagne, "Orderly structure in jet turbulence," J. Fluid Mech. 48, 547 (1971).

${ }^{3}$ K. Bremhorst and P. G. Hollis, "Velocity field of an axisymmetric pulsed, subsonic air jet," AIAA J. 28, 2043 (1990).

${ }^{4}$ P. J. Vermeulen, V. Ramesh, and W. K. Yu, "Measurement of entrainment by acoustically pulsed axisymmetric air jets," Trans. ASME: J. Eng. Gas Turbines Power 108, 479 (1986).

${ }^{5}$ H. Johari and V. Motevalli, "Flame length measurements of burning fuel puffs," Combust. Sci. Technol. 94, 229 (1993).

${ }^{6}$ J. C. Hermanson, R. Dugnani, and H. Johari, "Structure and flame length of fully-modulated, pulsed diffusion flames," Combust. Sci. Technol. 155, 203 (2000)

${ }^{7}$ J. M. Richards, "Puff motions in unstratified surroundings," J. Fluid Mech. 21, 97 (1965).
${ }^{8}$ L. S. G. Kovasznay, H. Fujita, and R. L. Lee, "Unsteady turbulent puffs," Adv. Geophys. 55, 253 (1974).

${ }^{9}$ K. Oshima, L. S. G. Kovasznay, and Y. Oshima, "Sound emission from burning puff," Lect. Notes Phys. 76, 219 (1977).

${ }^{10}$ A. Glezer and D. Coles, "An experiment study of a turbulent vortex ring," J. Fluid Mech. 211, 243 (1990).

${ }^{11}$ R. Sangras, O. C. Kwon, and G. M. Faeth, "Self-preserving properties of unsteady round nonbuoyant turbulent starting jets and puffs in still fluids," J. Heat Transfer 124, 460 (2002); F. J. Diez, R. Sangras, O. C. Kwon, and G. M. Faeth, "Erratum: Self-preserving properties of unsteady round nonbuoyant turbulent starting jets and puffs in still fluids," ibid. 125, 204(E) (2003).

${ }^{12}$ E. Ghaem-Maghami and H. Johari, "Concentration field measurements within isolated turbulent puffs," ASME Trans. J. Fluids Eng. 129, 194 (2007).

${ }^{13}$ M. Gharib, E. Rambod, and K. Shariff, "A universal time scale for vortex ring formation," J. Fluid Mech. 360, 121 (1998).

${ }^{14}$ F. Scarano and M. L. Riethmuller, "Iterative multigrid approach in PIV image processing with discrete window offset," Exp. Fluids 26, 513 (1999).

${ }^{15}$ I. J. Wygnanski and H. E. Fiedler, "Some measurements in the selfpreserving jet," J. Fluid Mech. 38, 577 (1969).

${ }^{16}$ H. B. Fischer, J. E. List, C. R. Koh, J. Imberger, and N. H. Brooks, Mixing in Inland and Coastal Waters (Academic, New York, 1979).

${ }^{17}$ H. J. Hussein, S. P. Capp, and W. K. George, "Velocity-measurements in a high-Reynolds-number, momentum-conserving, axisymmetrical, turbulent jet," J. Fluid Mech. 258, 31 (1994).

${ }^{18}$ B. J. Boersma, G. Brethouwer, and F. T. M. Nieuwstadt, "A numerical investigation on the effect of the inflow conditions on the self-similar region of a round jet," Phys. Fluids 10, 899 (1998).

${ }^{19}$ D. R. Webster, P. J. W. Roberts, and L. Ra'ad, "Simultaneous DPTV/PLIF measurements of a turbulent jet," Exp. Fluids 30, 65 (2001).

${ }^{20}$ N. R. Panchapakesan and J. L. Lumley, "Turbulence measurements in axisymmetric jets of air and helium. Part 1. Air jet," J. Fluid Mech. 246, 225 (1993).

${ }^{21}$ T. Maxworthy, "Turbulent vortex rings," J. Fluid Mech. 64, 227 (1974).

${ }^{22}$ D. Han and M. G. Mungal, "Direct measurement of entrainment in reacting/nonreacting turbulent jets," Combust. Flame 124, 370 (2001).

${ }^{23}$ A. M. Falcone and J. C. Cataldo, "Entrainment velocity in an axisymmetric turbulent jet," ASME Trans. J. Fluids Eng. 125, 620 (2003).

${ }^{24}$ J. C. Hermanson, E. Ghaem-Maghami, and H. Johari, "CO/UHC emissions of fully modulated diffusion flames," Combust. Sci. Technol. 176, 1855 (2004).

${ }^{25}$ J. C. Hermanson, H. Johari, D. P. Stocker, and U. G. Hegde, "Buoyancy effects in strongly-pulsed, turbulent diffusion flames," Combust. Flame 139, 61 (2004). 